\title{
Frequency and recency of reward and inferotemporal lesions'
}

\author{
MARTHA WILSON, LAWRENCE ROTHBLAT, AND EMILY \\ KIRSTEIN, DEPARTMENT OF PSYCHOLOGY, UNIVERSITY \\ OF CONNECTICUT, Storrs, Conn. 06268
}

The hypothesis that monkeys with inferotemporal lesions are deficient in remembering which cues in visual discrimination learning are rewarded was tested. Contrary to predictions, the performance of operated $S s$ was controlled by the frequency of reward while that of normal controls was a function of the recency of reward.

A short-term memory impairment has been described for monkeys with inferotemporal (IT) lesions by Wilson, Wilson, \& Sunenshine (in press). This interpretation of the IT deficit departs from recent descriptions which have stressed impairment in attending to various aspects of the stimulus (Butter, Mishkin, \& Rosvold, 1965; Iversen \& Weiskrantz, 1967). However, as the latter authors point out, their results could equally well be attributed to selective forgetting. The present study was designed as a direct test of the hypothesis that monkeys with IT lesions fail to remember which was the rewarded cue in visual discrimination learning. Furthermore, the design allowed a distinction to emerge between failing to establish a stimulus-reward association, and failing to remember this over succeeding trials.

Experimental design. An experimental design devised by Campione (1965), to test for transitivity of choices of rewarded stimuli, was used here. A series of problems was presented, each of which consisted of three training trials and one test trial. On the training trials, three stimuli, denoted $\mathrm{A}, \mathrm{B}$, and $\mathrm{C}$, were presented two at a time, as shown in Fig. 1. The stimulus represented on the left was rewarded. Over the three training trials, A was correct two times, $\mathrm{C}$ was incorrect two times, and $\mathrm{B}$ was correct once, and incorrect once. The test trial presented one of the three pairs of training stimuli with reward consistent with training, and provided information about the tendency of $S$ to choose between stimuli with different frequencies of reward. If frequency of reward influences choices in a transitive fashion, A should be chosen over $\mathrm{C}$ more often than $\mathrm{A}$ over $\mathrm{B}$, or $\mathrm{B}$ over $\mathrm{C}$.

It was also possible to see the effects of recency of training on the choice of stimuli, independent of the effects of frequency. As shown in Fig. 1, the ordinal position of the training trial which presented the same stimuli as the test trial was systematically varied. If $\mathrm{S}$ attempts to remember the correct stimulus of a pair, his choice may vary as a function of the recency of presentation of that pair.

The nine types of Experimental Problem shown represent combinations of the three levels of frequency and the three levels of recency which may contribute to performance on the test trials. Balancing the order of all the training trials produced 18 types of problem in all. Standard Problems, on which the stimuli remain constant on all trials of each problem, were also given.

These tests for the effects of frequency and recency of reward depend upon several assumptions. First, it must be assumed that the stimuli are adequate, i.e., that $S$ is attending to the relevant aspect of the stimulus. Secondly, it must be assumed that every trial is informative, i.e., that $\mathbf{S}$ infers that if one stimulus is not rewarded, the other one is, and conversely. In a preliminary experiment, using 12 naive intact monkeys as Ss, the same design failed to produce evidence for the effects of frequency of reward at more than a marginal level of significance. Therefore, in the present study, highly experienced Ss were used, in order to maximize the likelihood that Ss would attend to the relevant cues, and gain information on each trial.
Subjects. Six rhesus monkeys served as Ss. Three had sustained lesions of IT cortex approximately six months prior to the start of this experiment. Postmortem inspection of the operated brains showed that the ablations were confined to the middle and inferior temporal gyri with some additional superficial damage to the superior temporal gyrus.

Procedure. Ten problems were presented on each testing day, consisting of six Experimental Problems and four Standard Problems. Testing was continued for 12 days, with four replications of each three-day block of 18 Experimental Problems. Thus each of the nine types of Experimental Problems which differed in frequency and recency of reward, was given eight times. The order of presentation of the Experimental Problems was balanced within days and over days. The Standard Problems were given as Problems 1, 4,7 and 10 on a given day. Left-right position of reward and reward sequences for all problems were balanced within and over days.

The stimuli consisted of cut-out Masonite forms which differed in color (red, orange, yellow, green, blue, black and white) and shape (star, circle, cross, triangle, $T$, and square). Color was relevant on all problems, and form remained constant within and over trials of a given problem. Stimulus values were randomly assigned with two restrictions: the same color or form did not appear on successive problems, and particular stimulus values appeared equally often.

Ss were tested in a WGTA, fitted with a stimulus board having foodwells spaced 14 in. apart. Ss displaced one of the two stimuli from the foodwell to obtain a raisin reward. Noncorrection procedure was used throughout.

Results. Performance of the two groups on the Experimental and Standard Problems is shown in Table 1. Overall percentage correct on Experimental Problems did not differ for the two groups. Separate analyses of variance show that the IT Ss were affected significantly by the frequency of reward $(\mathrm{F}=19.77 \mathrm{df}=$ $2 / 4, p<.01)$. Their choices of $A$ over $C$ were more frequent than A over B, or B over C. They did not show transitivity of choice since $A$ was not preferred to $C$ significantly more than to $B$, as would have been the case if they had added up rewards and nonrewards algebraically, but it is clear that they preferred the most frequently rewarded stimulus. Recency of a given rewarded pair had no effect upon performance suggesting that the preference for $A$ was maintained over trials regardless of the degree of recency.

In contrast, the $\mathrm{NC}$ group showed a strong recency effect ( $\mathrm{F}=$ 10.80 , df $=2 / 4, p<.05$ ), but no effect of frequency. Their performance was equally good on all test pairs, and depended solely on how recent their experience with that pair had been.

On the Standard Problems, analysis of variance of Trials 2-4 showed that the IT were inferior in visual discrimination learning under standard conditions as would be expected $(\mathrm{F}=9.00, \mathrm{~d} f=$ $1 / 4, \mathrm{p}<.05)$.

Discussion. The results of this study do not support the hypothesis that IT monkeys have difficulty in establishing stimulus-reward associations, or in maintaining them over trials. On the contrary, they appeared to choose the most frequently rewarded stimulus more often than did the $\mathrm{NC}$ group, and to maintain this choice independently of what was most recently rewarded. In this respect, they resembled the naive normal $S s$ in the preliminary study referred to. The NC group appeared to forget the outcomes of previous trials and responded solely on the basis of the preceding trial. These two strategies produced identical overall performance on the Experimental Problems but on the Standard Problems, the ITs showed the expected visual discrimination learning impairment. It is not at all clear from these data why this should have been so. However, this experiment suggests that a simple loss-of-function

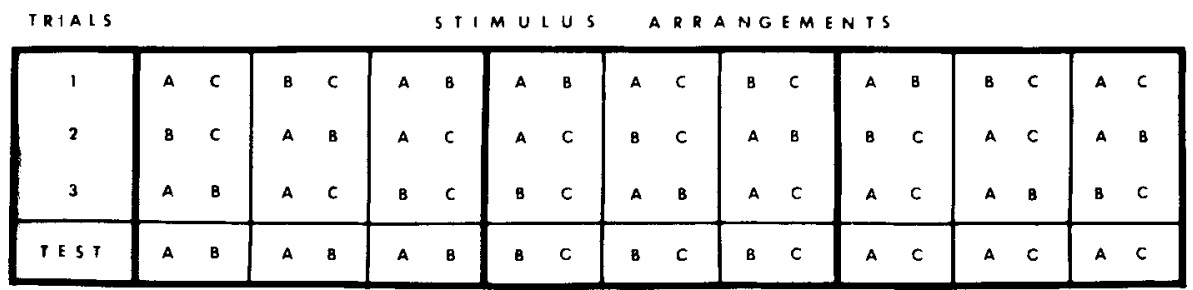

Fig. 1. 
Table 1

Percentage Correct Response on Test Trials as a Function of Frequency and Recency of Reward

\begin{tabular}{ccccccccc} 
& \multicolumn{4}{c}{ Test Trial } & & \multicolumn{3}{c}{ Recency ${ }^{\mathbf{2}}$} \\
${$\cline { 2 - 5 }$} }$ & AB & BC & AC & Standard & 1 & 2 & 3 \\
\hline NC & $61 \pm 05$ & $64 \pm 03$ & $64 \pm 12$ & $75 \pm 01$ & & $75 \pm 06$ & $58 \pm 02$ & $56 \pm 08$ \\
IT & $67 \pm 05$ & $53 \pm 03$ & $72 \pm 02$ & $66 \pm 04$ & $64 \pm 04$ & $65 \pm 06$ & $61 \pm 04$ \\
\hline
\end{tabular}

aProblems with the most recent identical training trial are denoted 1.

explanation, such as an impairment in memory, is not adequate. More fundamental descriptions of how visual information is processed in naive and sophisticated normal and IT monkeys is needed to explain those situations in which ITs display deficits in associative learning, and those in which they do not (Wilson, in press)

\section{REFERENCES}

BUTLER, C. M., MISHKIN, M., \& ROSVOLD, H. E. Stimulus generalization in monkeys with inferotemporal and lateral occipital lesions. In D. J. Mostofsky (Ed.), Stimulus generalization. Stanford, Calif.: Stanford Univ. Press, 1965. Pp. 119-133.

CAMPIONE, J. C. Transitivity and choice behavior. Unpublished doctoral dissertation. University of Connecticut, 1965.

IVERSEN, S., \& WEISKRANTZ, L. Perception of redundant cues by monkeys with inferotemporal lesions. Nature, 1967, 214, 241-243.

WILSON, M. Inferotemporal cortex and the processing of visual information in monkeys. Neuropsychologia, 1968, 6, 135-140.

WILSON, M., WILSON, W. A., Jr.; \& SUNENSHINE, H. S. Perception, learning and retention of visual stimuli by monkeys with inferotemporal lesions. J. comp. physiol. Psychol, in press.

\section{NOTE}

1. This experiment was supported in part by Grant MH 10972 from the National Institute of Mental Health, USPHS. 